\title{
Oncogenic role of the ubiquitin ligase subunit Skp2 in human breast cancer
}

\author{
Sabina Signoretti, ${ }^{1,2}$ Lucia Di Marcotullio, ${ }^{3}$ Andrea Richardson, ${ }^{1,2}$ Sridhar Ramaswamy, ${ }^{1,4}$ \\ Beth Isaac, ${ }^{1}$ Montserrat Rue,${ }^{5}$ Franco Monti, ${ }^{6}$ Massimo Loda, ${ }^{1,2}$ and Michele Pagano ${ }^{3}$ \\ ${ }^{1}$ Department of Medical Oncology, Dana Farber Cancer Institute, Harvard Medical School, Boston, Massachusetts, USA \\ ${ }^{2}$ Department of Pathology, Brigham \& Women's Hospital, Harvard Medical School, Boston, Massachusetts, USA \\ ${ }^{3}$ Department of Pathology, and New York University Cancer Institute, New York University School of Medicine, New York, \\ New York, USA \\ ${ }^{4}$ Whitehead Institute/MIT Center for Genomic Research, Cambridge, Massachusetts, USA \\ ${ }^{5}$ Department of Biostatistics, Dana Farber Cancer Institute, Harvard Medical School, Boston, Massachusetts, USA \\ ${ }^{6}$ Department of Oncology Azienda Unità Sanitaria Locale Rimini, and Istituto Oncologico Romagnolo, Rimini, Italy
}

\begin{abstract}
Estrogen receptor (ER) expression and Her-2 amplification define specific subsets of breast tumors for which specific therapies exist. The S-phase kinase-associated protein Skp2 is required for the ubiquitin-mediated degradation of the cdk-inhibitor p27 and is a bona fide proto-oncoprotein. Using microarray analysis and immunohistochemistry, we determined that higher levels of Skp2 are present more frequently in ER-negative tumors than in ER-positive cases. Interestingly, the subset of ER-negative breast carcinomas overexpressing Skp2 are also characterized by high tumor grade, negativity for Her-2, basal-like phenotype, high expression of certain cell cycle regulatory genes, and low levels of $\mathrm{p} 27$ protein. We also found that Skp2 expression is cell adhesion-dependent in normal human mammary epithelial cells but not in breast cancer cells and that an inhibition of Skp2 induces a decrease of adhesion-independent growth in both ER-positive and ER-negative cancer cells. Finally, forced expression of Skp2 abolished effects of antiestrogens, suggesting that deregulated Skp2 expression might play a role in the development of resistance to antiestrogens. We conclude that Skp2 has oncogenic potential in breast epithelial cells and is overexpressed in a subset of breast carcinomas (ER- and Her-2 negative) for which Skp2 inhibitors may represent a valid therapeutic option.
\end{abstract}

J. Clin. Invest. 110:633-641 (2002). doi:10.1172/JCI200215795.

\section{Introduction}

Patients with breast carcinomas can display a different response to therapy and clinical outcome despite similar stage and grade of disease. Gene expression profiling using microarray technology has recently allowed the identification of subgroups of breast carcinomas with distinct molecular signatures and biologic behavior. Targeted therapy with tamoxifen (1) and herceptin (2) is available for estrogen receptor-positive (ER-positive) and Her-2-overexpressing breast tumors, respectively. ER-negative breast carcinomas have been further classified by molecular profiling into at least two biologically different subtypes. These include Her2-positive as well as Her-2-negative tumors with a basal-like phenotype $(3,4)$. This latter group of ERnegative, Her-2-negative tumors expressing genes

Received for publication April 25, 2002, and accepted in revised form June 25, 2002.

Address correspondence to: Michele Pagano, Department of Pathology, and New York University Cancer Institute, New York University School of Medicine, 550 First Avenue, MSB 599, New York, New York 10016, USA. Phone: (212) 263-5332; Fax (212) 263-5107; E-mail: paganm02@med.nyu.edu. Conflict of interest: No conflict of interest has been declared. Nonstandard abbreviations used: estrogen receptor (ER); F-box proteins (Fbp's); S-phase kinase-associated protein 1 (Skp1); human breast ductal epithelial cells (HBECs);

bromodeoxyuridine (BrdU); progesterone receptors (PR); proliferating cell nuclear antigen (PCNA). associated with a basal-like phenotype typically shows resistance to standard treatments and is characterized by aggressive biologic behavior.

F-box proteins (Fbp's) are characterized by an approximately 40-amino acid domain called F-box because it was first identified in cyclin F (5). Studies involving several organisms have shown that Fbp's play a crucial role in the ubiquitin-mediated degradation of cellular regulatory proteins (e.g., cyclins, cdk-inhibitors, $\beta$-catenin, IKB, etc.) (reviewed in refs. 6-8). Indeed, Fbp's are subunits of ubiquitin ligases named SCFs because they are formed by the following basic subunits: S-phase kinaseassociated protein 1 (Skp1), Cul1, Roc1/Rbx1, and one of many Fbp's. Since the substrate specificity of SCF ligases is dictated by different Fbp's that act as substrate-targeting subunits, a large number of Fbp's is necessary to ensure highly specific substrate recognition. In fact, large families of Fbp's are present in all eukaryotes (e.g., 11 members in yeast, 29 members in flies, and at least 54 in mammals; refs. 9-11).

Human Skp1 and the F-box protein Skp2 were originally identified as two proteins physically interacting with cyclin A and therefore were designated as S-phase kinase-associated proteins (12). We and others have demonstrated that Skp2 is required for the ubiquitination and consequent degradation of the $\mathrm{cdk}$ inhibitor p27 both in vivo $(13,14)$ and in vitro $(13,15)$. Skp2-deficient mice grow slower than littermate con- 
trols and show smaller organs, with all tissues containing decreased numbers of cells (16). Skp2 $2^{-/-}$cells show high levels of $\mathrm{p} 27$, polyploidy, and centrosome overduplication. This phenotype underscores the importance of Skp2 in positively regulating cell proliferation. All cellular and histopathological abnormalities observed in Skp2-deficient mice are abolished in Skp2/p27 double-knockout mice, indicating that p27 is a primary substrate of Skp2.

A decrease in the level of $\mathrm{p} 27$ protein in the absence of alteration of the p27 gene is commonly seen in many human cancers, including epithelial cancers, brain tumors, and lymphoproliferative neoplasms (reviewed in ref. 17). Importantly, reduced levels of p27 correlate with poor prognosis. In colon cancers (18), non-small cell lung cancers (19), oral squamous cell carcinomas (20), lymphomas (21), and astrocytic brain tumors (22), this decrease in p27 protein levels has been shown to be due to an increase in its ubiquitinproteasome-mediated proteolysis. One possible reason for this enhanced degradation might lie in the presence of high levels of Skp2. Accordingly, it has been shown that unregulated Skp2 expression induces p27 degradation and entry into S-phase, both in the presence of low serum concentration (14) and in the absence of cell adhesion (23). Indeed, it has been shown recently that Skp2 has oncogenic potential (24, $25)$ and is overexpressed in oral epithelial $(20,24)$, colorectal carcinomas (26), and lymphomas $(25,27)$.

Because p27 is downregulated in aggressive human breast cancers (28-30), and because of the role of Skp2 in p27 degradation, we have assessed the levels of Skp2 and p27 in human breast cancer samples and performed experiments to evaluate the role of Skp2 in breast cancer cell lines. Our data support an oncogenic role for Skp2 and suggest it may be a therapeutic target in a subset of ER-negative, Her-2-negative human breast cancers with a basal-like phenotype that is typically resistant to standard therapies.

\section{Methods}

Patient populations. In sample set A (oligonucleotide microarray analysis), a total of 90 primary, untreated, breast carcinoma frozen tissue samples were selected from a repository held by the Dana-Farber/Harvard Specialized Program of Research Excellence in Breast Cancer. Histologic sections of tumor were blindly reviewed and scored for a modified Bloom-Richardson grade. Hormone receptor and Her-2 receptor immunohistochemical sections (performed for clinical assessment) were reviewed and scored as positive or negative. For sample set B (immunohistochemistry and immunofluorescence analysis), breast cancer specimens from the years 1987-1996 were retrieved from the files of the Department of Oncology Azienda Unità Sanitaria Locale, Rimini, Italy. Eighty-four patients with breast carcinoma were selected for the study. All the patients developed either local recurrences or metastatic disease (nodal or distant) during the follow-up period. Stage and grade were available in all cases. The median followup for survivor patients was 40 months.

Oligonucleotide microarray analysis. The frozen bulk tumor samples (sample set A) were confirmed to contain at least $70 \%$ viable tumor by area on histologic thin sections. Total RNA extracted from frozen tissue was used to generate biotinylated cRNA target and subsequently hybridized to Affymetrix U95A oligonucleotide probe arrays (Affymetrix Inc., Santa Clara, California, USA), according to standard protocols (31). Raw expression values were obtained using GENECHIP software from Affymetrix and further analyzed using the DNA-Chip Analyzer (dChip) custom software. Of the 90 arrayed samples, one array was excluded for a gross defect (scratch), leaving 89 tumors for analysis. Hierarchical clustering was performed using the dChip software. The clustering diagram in Figure 1a was performed using a subset of 2,130 genes filtered for those showing a variation of expression across the samples. The filtering criteria included genes with a coefficient of variation (SD/mean) between 0.65 and 10.0 and with a mean intensity value of greater than 50 units in at least $5 \%$ of the tumor samples. The smaller clustering diagram in Figure $1 \mathrm{~b}$ was performed using a smaller set of 41 genes selected to show the correlation between the expression of Skp2, cell cycle regulatory genes, hormone receptors and related genes, Her-2 amplicon genes, keratin genes, and several markers of the basal cell phenotype.

Immunohistochemistry and immunofluorescence. Both p27 and Skp2 expression was assessed in all 84 tumor samples (sample set B), while Ki67 expression was investigated in a subset of 37 tumors. Immunohistochemistry experiments were performed as described previously (32). The following primary Ab's were used: anti-Skp2 (Zymed Inc., South San Francisco, California, USA) at 1:100 dilution as described (25), anti-p27 (Transduction Laboratories, Lexington, Kentucky, USA) at 1:200 dilution, anti-Ki67 (Immunotech, Westbrook, Maine, USA) at 1:50 dilution, anti-ER (DAKO Corp., Carpinteria, California, USA) at 1:100 dilution, and anti-Her-2 (AO485; DAKO Corp.) at 1:200 dilution.

Scoring of immunostained slides was done according to the percentage of tumor cells exhibiting nuclear (p27, Ki67, and ER) or nuclear/cytoplasmic staining (Skp2). To define high and low p27 expressors we used a cutoff of $50 \%$ or more positive tumor cells, which is the cutoff used in the majority of the previous studies. To select a cutoff for Skp2 we tried first the mean and the median. Because the majority of patients had low levels of Skp2, using either the mean or the median as a cutoff would have included cases with a low percentage of positive cells in the high expressor category. To have a more meaningful classification, we established the cutoff in the 75 th percentile (10\% or more of positive cells). A cutoff of $10 \%$ or more was used to define ER-positive cases. Fisher's exact test was used to assess the association between Skp2 and p27 expression levels. Linear regression analysis was used to assess the association between Skp2 and Ki67 expres- 
a

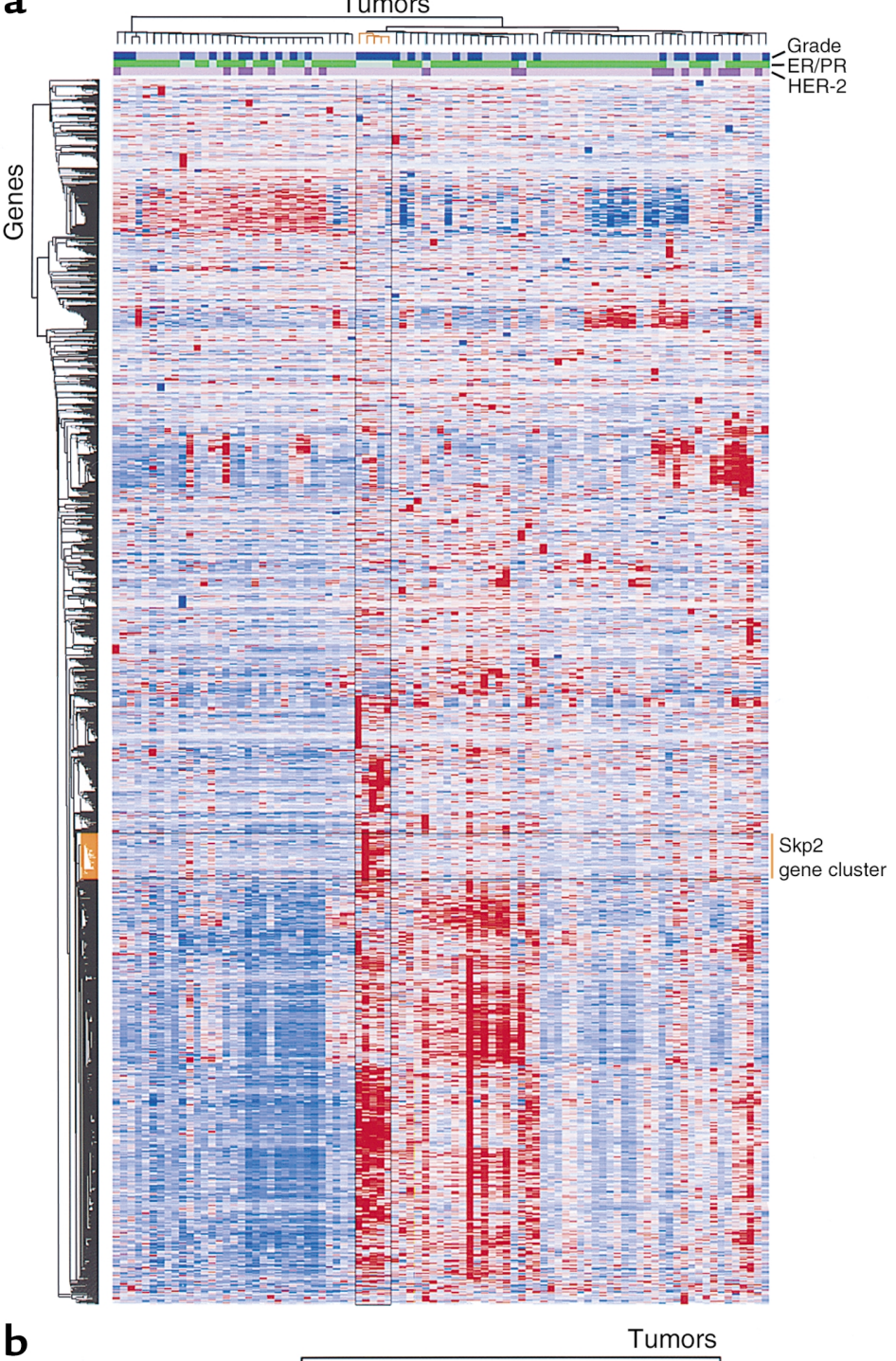

Figure 1

Hierarchical clustering of 89 breast tumor samples (sample set A). Each column represents a tumor sample, and each row represents a different gene probe set. The relative expression level of the genes in the samples is indicated by a gradient of color, with blue representing expression levels below the mean and red representing expression levels above the mean. The tumors are clustered in the dendrogram at the top, and the genes are clustered in the dendrogram down the side. Tumors and genes appearing next to each other are more similar in expression pattern. The pathologic data for each tumor sample is represented by the color bars below the tumor dendrograms: dark blue, modified Bloom-Richardson grade III; light blue, modified Bloom-Richardson grade I or II; dark green, ER and PR positive; light green, ER and PR negative; dark purple, Her-2 overexpressed (3+); light purple, Her-2 nonoverexpressed. The tumor clusters showing the highest levels of Skp2 expression and the gene clusters that include the $S k p 2$ gene are highlighted in orange. (a) Hierarchical clustering of 89 tumors for 2,130 filtered genes. (b) Clustering of 89 tumors to demonstrate relative expression of 41 selected genes, including cell cycle regulatory genes (e.g., cyclins, Cdks, Cks1, Cks2, PCNA, p16), keratins, hormone receptors (e.g., ER $\alpha$, ER $\beta$, ERR $\alpha$, androgen receptor), ER-associated genes (e.g., HNF3, TFF3, GATA3, LIV-1), Her-2 amplicon genes (e.g., Her-2, PPAR-binding protein, MLN64, GRB7), and genes associated with the basal-like tumor subgroup (e.g., UDP-N-Ac-galactosamine, chitinase 3-like 2, P-cadherin, AC133/prominin-like 1, HDGF).

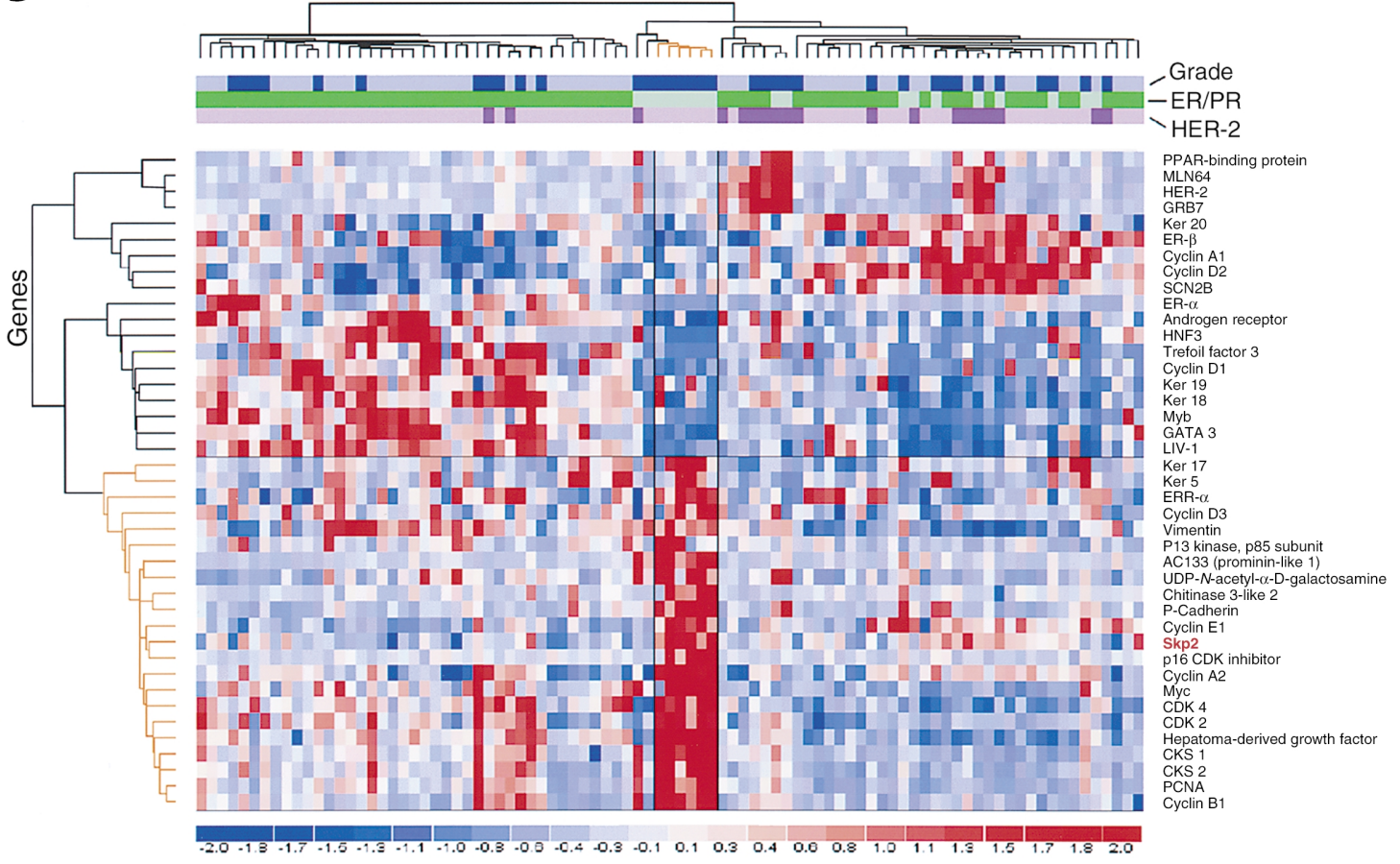


sion levels. Stage and grade of the tumors were compared with Skp2 and p27 dichotomized expression levels using Fisher's exact test. The proportional hazards model was used to assess the association of Skp2 and p27 with survival time, controlling for stage and grade of the tumor.

Both the breast carcinoma cell line MCF-7 (American Type Culture Collection, Rockville, Maryland, USA) and five paraffin-embedded breast carcinoma samples from sample set $B$ were submitted to doubleimmunofluorescence staining for Skp2/Ki67. Doubleimmunofluorescence experiments were performed as described previously (33).

Cell cultures, cell synchronization, and cell cycle analysis. Human breast cancer cell lines (MCF-7 and MDAMB-435), human breast cell line HBL-100 (expressing SV-40 large $T$ protein), human fibrosarcoma cell line HS-913-T, and human embryonic kidney adenovirusand SV-40 large T-transformed epithelial cell line 293 were obtained from the American Type Culture Collection and cultured as described (34). Normal human breast ductal epithelial cells (HBECs) were obtained from Clonetics/BioWhittaker Inc. (Walkersville, Maryland, USA) and cultured in the growth medium recommended by the manufacturer as described (34). Human normal diploid fibroblasts (HF, also called IMR-90) and SV-40 large T-transformed IMR-90 (IDH-4) were grown as described (34).

To test the effects of lack of adhesion to the ECM, cells were trypsinized and reseeded on Petri dishes coated with $1 \%$ agarose (suspension) using $0.5 \times 10^{6}$ to $1 \times 10^{6}$ cells per $10-\mathrm{cm}$ dish. Colony formation in soft agar was done as described (23). For cell cycle analyses, adherent cells were grown on glass coverslips, labeled for 5 hours with $10 \mu \mathrm{m}$ bromodeoxyuridine (BrdU), rinsed with PBS, and fixed for 10 minutes in $-20^{\circ} \mathrm{C}$ methanol-acetone (1:1). Fixed cells were stained for BrdU and quantified as described previously (35).

Retroviral-mediated gene transfer. Packaging GP-293 cells (CLONTECH Laboratories Inc., Palo Alto, California, USA) were transfected with retroviral plasmids according to the manufacturer's instructions. Fortyeight hours after transfection, the virus-containing medium was collected and supplemented with 8 $\mu \mathrm{g} / \mathrm{ml}$ polybrene (Sigma-Aldrich, St. Louis, Missouri, USA). Then, the culture medium of the target cells was replaced with this viral supernatant for 24 hours. After a 12-hour recovery in normal medium, the infection process was repeated a second time with Skp2, cyclin $\mathrm{D} 1$, and cyclin $\mathrm{E}$ viruses, and three to four times with Skp2 $(\Delta \mathrm{F})$ virus. The percentage of infected cells was quantified by flow cytometry or immunofluorescence. In all cases, greater than $85 \%$ of the cells were infected. Multiple genes were introduced sequentially.

\section{Results}

Skp2 message is highly expressed in a subgroup of ER-negative and Her-2-negative tumors by oligonucleotide microarray analysis. The 89 arrayed primary breast tumor sam- ples (sample set A) were first analyzed by hierarchical clustering using a filtered set of 2,130 variably expressed genes (Figure 1a). The 89 tumors are clustered across the top and the genes are clustered down the side, based on similar patterns of expression. The gene cluster that includes Skp2 is highlighted in orange in the horizontal dendrogram. The tumor cluster that shows the highest levels of expression of Skp 2 is highlighted in orange in the vertical dendrogram. The histologic tumor grade and the results of the immunohistochemistry analysis, ERs and progesterone receptors (ERs/PRs) and Her-2 proteins, are represented by the blue, green, and purple color bars, respectively. First, higher levels of Skp2 mRNA (>0.3fold over the mean expression level) were more frequently observed in ER-negative tumors (11 of 18 or $61 \%$ ) than in ER-positive cases ( 16 of 71 or $23 \%$ ). Second, as shown in Figure 1a, the highest Skp2 expression levels were in a small subgroup of five tumors that were all high grade, negative for ERs/PRs, and negative for Her-2 overexpression. When compared with the other samples, the tumor subset with high Skp2 expression was noted to have higher relative expression of other proliferation-associated genes and a number of genes described previously to characterize a basal-like subset of breast tumors $(3,4)$. To better visualize the correlation between the expression of some of these genes, we performed cluster analysis of the 89 tumors in a more limited gene space of 41 genes (Figure 1b). The 41 genes were selected to include Skp2, other cell cycle regulatory genes, hormone receptor genes, ER-regulated and ER-associated genes, genes from the Her-2 amplicon, keratins, and representative genes associated with the previously described basal-like tumor subgroup. In this more limited and selected gene space, there were now six tumors in the cluster characterized by high Skp2 expression (cluster highlighted in orange). The high Skp2 cluster included the 5 ER-, PR-, and Her-2-negative, high-grade cases that previously clustered together in the larger gene space, plus an additional ER/PR-negative, Her-2-negative, high-grade tumor. As shown in Figure 1b, the high-Skp2 tumor cluster was characterized by high expression of other genes encoding proteins known to physically interact with Skp2: cyclin $\mathrm{A} 2$, cyclin $\mathrm{E} 1, \mathrm{Cdk} 2$, and Cks1. In addition, markers of S-phase, such as proliferating cell nuclear antigen (PCNA), and G2/M, such as cyclin B1 and Cks2, were also high in this subgroup. Not all cyclins, however, clustered with Skp2. For example, cyclin D1 and cyclin D2, two cyclins known to play an important role for the proliferation of breast epithelium, were not highly expressed in this subset. In contrast, cyclin D3 and Skp2 showed a similar expression pattern. Finally, the tumor subset with high Skp2 levels also showed expression of genes characteristic of the basal-like breast cancer subgroup described by Sorlie et al. (4), including keratin 5, keratin 17, UDP- $N$-Acgalactosamine, chitinase 3 -like 2 , and P-cadherin. 
High levels of Skp2 protein are associated with ER negativity, low expression of $\mathrm{p} 27$, high proliferation rate, and poor survival. We then compared the results of the microarray analysis with an immunohistochemical study in a separate series of 84 breast carcinomas (sample set B), for which follow-up data were also available. We analyzed levels of Skp2, p27, Ki67, and ER proteins. Skp2positive cells ranged from $0 \%$ to $35 \%$ (median $4 \%$ ) in the breast carcinoma samples (Figure 2g), whereas normal breast epithelium was consistently negative for Skp2 expression (Figure 2, b,d, and f). Expression levels ranged from $0 \%$ to $90 \%$ (median $40 \%$ ) for $\mathrm{p} 27,0 \%$ to $70 \%$ (median 15\%) for Ki67, and 0\% to $90 \%$ for ER. When cutoff points were used to categorize high and low expressors, breast carcinoma cases showed three major patterns of Skp2/p27 expression (Figure $2 \mathrm{~g}$ ). Thirty tumors (36\%) were characterized by high p27 and low Skp2 levels. Twenty-one tumors (25\%) expressed low p27 and high Skp2 levels. In 32 tumors (38\%), both low p27 and low Skp2 levels were observed. Only one tumor expressed both high p27 and high Skp2 levels. Fisher's exact test showed that the percentage of high Skp2 expressors was significantly greater in the group with low p27 expression (37\%) than in the group with high p27 expression (3\%) $(P<0.001)$. In addition, we found that high levels of Skp2 protein were more frequently observed in ERnegative tumors (12 of 18 or $67 \%$ ) than in ER-positive cases ( 7 of 47 or $15 \%$ ) (Fisher's exact test, $P<0.0001$ ) and in tumors with high proliferation index (Ki67 positive) (Figure 3, a-d). Simple regression analysis demonstrated a direct linear association between Skp2 and Ki67 expression levels $\left(R^{2}=0.84\right)$ (Figure 3e). We further investigated whether Skp2 and Ki67 proteins were expressed in the same subset of tumor cells. To this end, double-immunofluorescence experiments for Skp2 and Ki67 were performed both in breast cancer MCF-7 cells and in paraffin-embedded tumor samples. In both cases, $\mathrm{Skp} 2$ protein was detected only in cells expressing Ki67 (Figure 3, f-k). Both Ki67 and Skp2 were localized in the nucleus. A subset of cells that expressed Ki67 protein was negative for Skp2 expression, indicating that, as already shown for lymphomas (27), levels of Skp2 might define the fraction of Ki67 cells that are in S-phase.

Finally, we looked at the relationship between Skp2 and survival. There was a statistically significant association between Skp2 expression levels and grade (Fisher's exact test, $P<0.001$ ). The Cox regression model showed that high expression levels of Skp2 were associated with poor survival (calculated from the time of the first recurrence) in the univariate model [hazard ratio $=2.83,95 \%$ confidence interval $=(1.48-5.44)$, $P=0.02]$. When grade was added to this model, Skp2 still showed a trend toward discrimination of biologic behavior [ (hazard ratio $=1.71,95 \%$ confidence interval $=(0.83-3.50)]$, although this was not statistically significant. As expected, high values of p27 expression were associated with better survival [hazard ratio $=$
$0.37,95 \%$ confidence interval $=(0.16-0.84), P=0.018]$ in the univariate analysis. When stage, grade, or Skp2 were added into the model one at a time, similar to Skp2, p27 also showed a trend in predicting survival (hazard ratio $=0.5$ ) but was no longer statistically significant. These results indicate that larger number of patients, with more statistical power, need to be interrogated to establish if Skp2 is an independent predictor of biologic behavior.

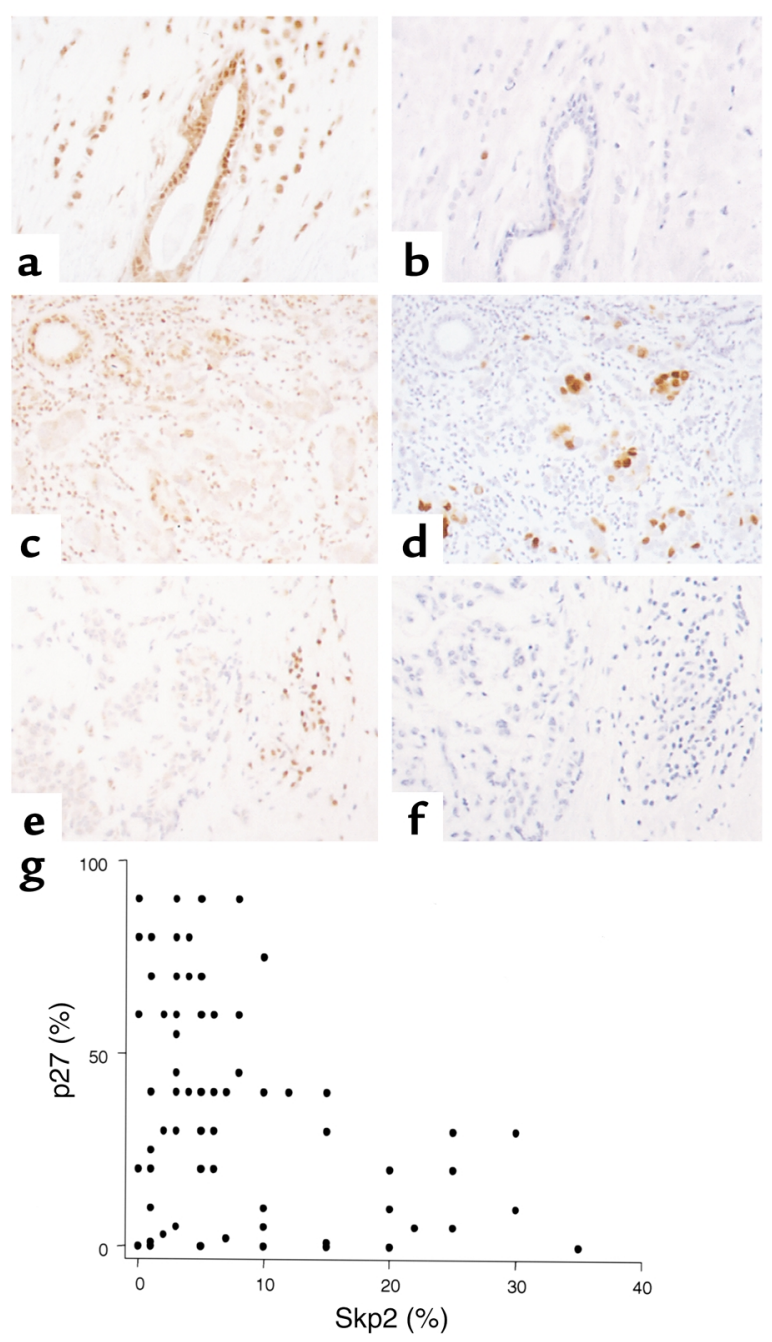

Figure 2

Relationship between Skp2 and p27 expression in 84 human breast carcinomas (sample set B). (a-f) Immunohistochemical stains for p27 (a, c, and $\mathbf{e}$ ) and Skp2 (b, d, and $\mathbf{f}$ ) of three breast carcinomas representative of the three patterns of Skp2/p27 expression. The carcinoma in the top row shows high p27 expression in both the residual normal duct in the center and in the infiltrating tumor surrounding it (a). Only one Skp2-positive cell is seen in the corresponding section in $\mathbf{b}$. The tumor in the middle panel shows a 27 -negative tumor (with positive residual normal ducts) (c). Strongly positive Skp2 tumor cells are seen in d. (e) Tumor that is negative for p27 (positive infiltrating lymphocytes are seen on the right). This tumor is also negative for Skp2 (f). (g) Graphic representation of the expression of Skp2 and p27 in the 84 tumors. Using the cutoffs (50\% for p 27 and $10 \%$ for Skp2) to define high and low expressors, tumors fall into three categories: high p27/low Skp2, low p27/high Skp2, and low p27/low Skp2. 

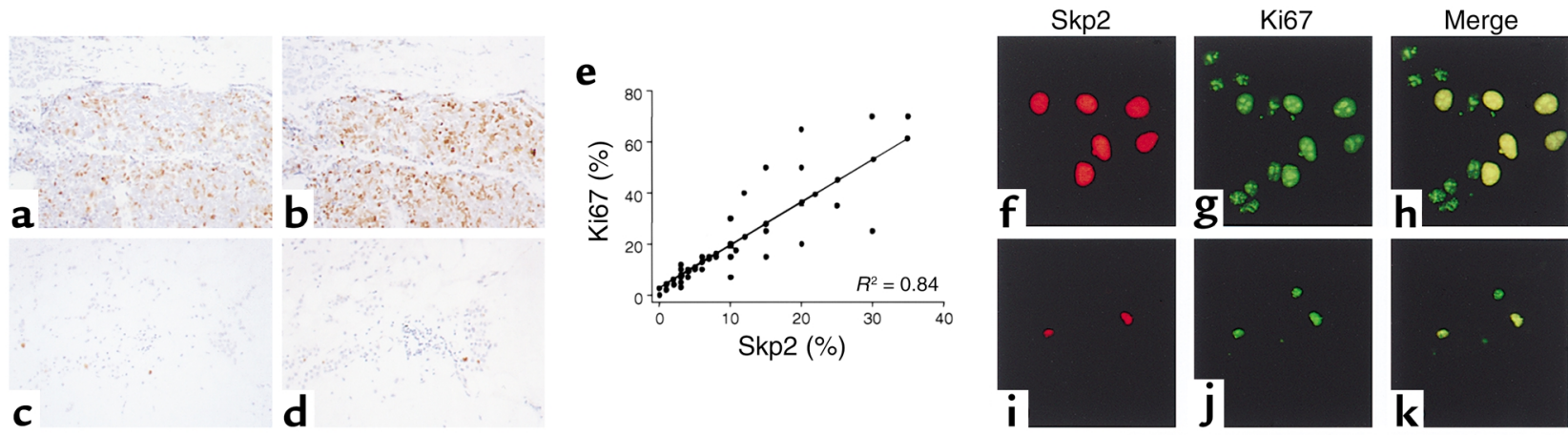

Figure 3

Correlation between Skp2 and proliferative index in sample set B. (a-d) Immunohistochemical stains of two representative breast carcinomas with anti-Skp2 ( $\mathbf{a}$ and $\mathbf{c}$ ) and anti-Ki67 (b and $\mathbf{d}$ ) Ab's. The tumor in the top row shows high Skp2/high Ki-67 levels. The one in the bottom row displays low Skp2/low Ki67 levels. (e) Graphic representation of the direct correlation between Skp2 expression and proliferative index in the 37 breast carcinomas. (f-k) Dual immunofluorescence of Skp2 with Ki67 in the breast cancer cell line MCF7 and in breast carcinomas. In MCF-7 cells (f, $\mathbf{g}$, and $\mathbf{h}$ ) as well as in breast carcinoma samples (i, j, and $\mathbf{k})$, Skp2 protein (Texas Red) was detected only in cells expressing Ki67 (FITC). Both Ki67 and Skp2 are localized in the nucleus. A subset of cells that expressed Ki67 protein was negative for Skp2 expression (h and $\mathbf{k})$.

Skp2 is required for anchorage independence in breast cancer cells. The results in human breast cancers suggest that high Skp2 expression might contribute to oncogenesis in breast epithelium. We have shown recently that in primary diploid fibroblasts Skp2 expression requires cell adhesion to the ECM (23) (see also Figure $4 \mathrm{~b}$, lanes 1-3). We determined whether the requirement of cell adhesion for the expression of Skp2 is also present in breast epithelial cells. Monolayer cultures of normal HBECs were trypsinized and replated in suspension. Under these conditions, the overall amount of Skp2 dramatically decreased by 12 hours, which corresponded to an accumulation of p27 (Figure 4a, lanes 1-3) and a cell cycle arrest (not shown). In contrast, MCF-7 cells (ER-positive), two ER-negative breast cell lines (HBL-100 and MDM-MB-435), and other transformed cell lines expressed the same levels of Skp2 and p27 and proliferated regardless of their adhesion conditions (Figure 4, a and b). Thus, transformed cell lines have lost an adhesion-dependent control of Skp2 that is still present in normal cells. To determine if this deregulation of $\mathrm{Skp} 2$ expression is linked to the transformed phenotype of cancer cells, we infected MCF-7

\section{Figure 4}

Expression of Skp2 is required for anchorage independence in breast epithelial cancer cells. (a) Proliferating normal HBECs (lanes 1-3), three different breast cell lines (lanes 4-12), (b) normal human fibroblasts (lanes 1-3), and other transformed cell lines (lanes 4-12) were trypsinized and replated in suspension. Cells were collected at the indicated times, and protein extracts were analyzed by immunoblotting with Ab's to the indicated proteins. (c) Comparative analysis of the number of colonies larger than $200 \mu \mathrm{M}$ in diameter obtained growing breast cancer cells infected with various retroviruses in soft-agar. A total of $10^{5}$ MCF- 7 cells or $5 \times 10^{3}$ MDM-MB- 435 cells were seeded in $0.3 \%$ agar supplemented in $5 \%$ calf serum. Colonies were scored after 3 weeks of growth. The number of colonies scored represented at least three independent experiments \pm the SD of the mean. and MDM-MB-435 cells with a retroviral vector expressing either Skp2 or a Skp2 dominant-negative mutant missing the F-box $[\operatorname{Skp} 2(\Delta \mathrm{F})](13)$. Infected cells were then monitored for their ability to grow in an anchorage-independent manner by forming colonies in soft agar. While Skp2 expression did not have any significant effect, the expression of $\operatorname{Skp} 2(\Delta \mathrm{F})$ mutant strongly inhibited the growth in soft agar of these two

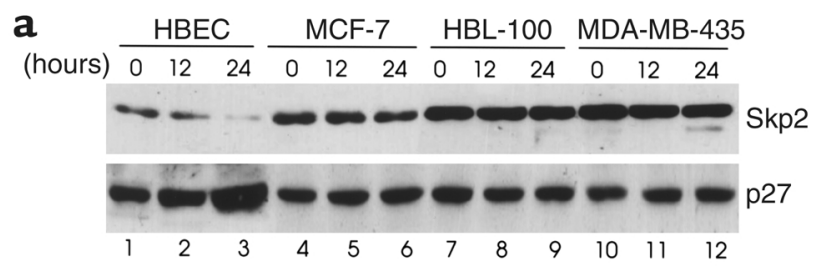

b

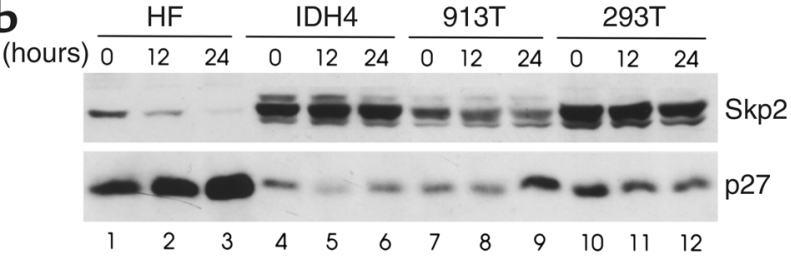

C

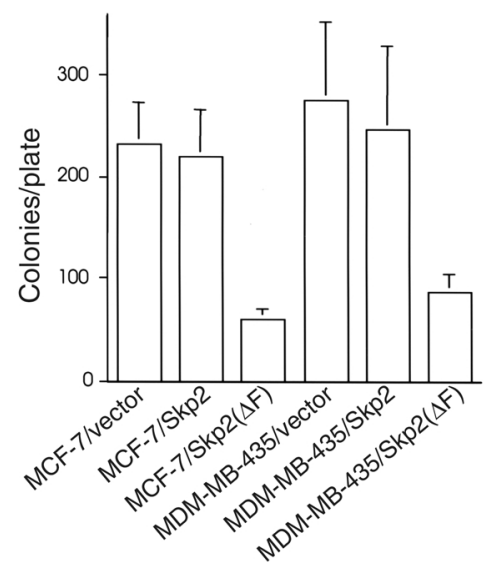


cell lines (Figure 4c). These results show that the inhibition of Skp2, in both ER-positive and ER-negative cells, results in a suppression of tumor growth and suggest that Skp2 is a target of therapies aimed at inhibiting the growth of breast carcinomas.

Skp2 expression abrogates antiestrogen-mediated cell cycle arrest in hormone-dependent breast epithelial cancer cells. Although most ER-negative tumors display higher levels of Skp2 (67\% as determined by immunohistochemistry and $61 \%$ by microarray), a fraction of ER-positive tumors also has higher Skp2 expression (15\% as determined by immunohistochemistry and $23 \%$ by microarray). This finding prompted us to study the relationship between estrogens and Skp2 expression. Thus, we assessed the estrogen-mediated cell cycle regulation of Skp2 in the well-characterized human breast MCF-7 cell line, which is estrogen sensitive. Asynchronous MCF-7 cells were arrested in G1 by treatment with tamoxifen combined with estrogen deprivation, as monitored by the downregulation of cyclin A levels and Cdk2 activity (Figure 5a, third panel from the top and bottom panel, respectively, lanes 1-3). Similar to cyclin A, the total abundance of Skp2 significantly decreased during cell cycle exit in parallel with an increase in p27 abundance (Figure 5a, top two panels, lanes 1-3). When cells were restimulated to enter the cell cycle by the sole addition of 17- $\beta$ estradiol, Skp 2 protein abundance increased in parallel with a decrease in p27 levels (Figure 5a, top two panels, lanes 4-6). In contrast, levels of cyclin D1 (Figure 5a, third panel from the top) and cyclin $\mathrm{E}$ (data not shown) did not change significantly in tamoxifen- and estradiol-treated cells. Thus, estrogens acting as mitogens in MCF-7 cells induce cell cycle re-entry and consequently Skp2 upregulation.

We then studied the phenotype of MCF-7 cells constitutively expressing either Skp2, cyclin D1, or cyclin E singularly, or Skp2 and cyclin D1 simultaneously. To express these proteins in MCF-7 cells, we used retroviral expression vectors and found that Skp2, cyclin D1, and cyclin $\mathrm{E}$ were all expressed in an estradiol- and tamoxifen-independent manner at levels two- to fourfold higher than those of the respective endogenous proteins (data not shown). Infected cells were either left asynchronous or were synchronized in G1 by tamoxifen treatment and estrogen deprivation. Percentage of cells in S-phase was then analyzed by BrdU incorporation (Figure 5b). No significant differences in BrdU incorporation were observed in untreated cells infected with either Skp2 or G1 cyclins. In contrast, significant differences were observed in tamoxifen-treated cells. In fact, compared with control cells, a significant increase in BrdU incorporation was observed in Skp2-expressing cells. In agreement with previous published results, a significant but more modest effect was also observed in cyclin D1-expressing cells, but not in cyclin E-expressing cells. These results show that the enforced expression of Skp2 in hormone-dependent breast cancer cells confer resistance to a G1 arrest mediated by antiestrogens and estrogen deprivation. Thus, in ER-positive breast carcinomas treated with antiestrogens, deregulated Skp2 expression might play a relevant role in the development of resistance to these drugs.

\section{Discussion}

This study provides insights into the oncogenic role of Skp2 in human breast cancer. Using microarray hybridization of RNA from 89 breast carcinomas (sample set A), we found that Skp2 message is overexpressed
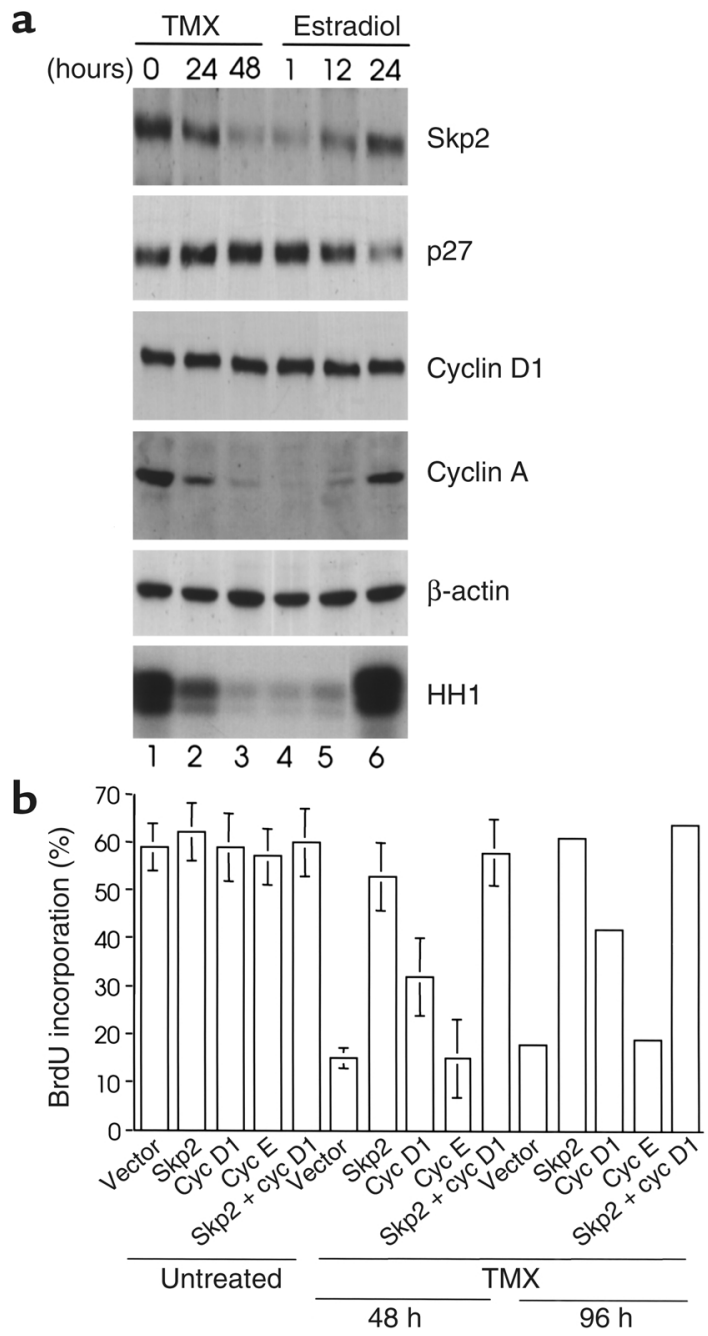

Figure 5

Enforced expression of Skp2 abrogates antiestrogen-mediated cell cycle arrest in MCF-7 cells. (a) MCF-7 breast cancer cells were synchronized in $\mathrm{G} 1$ phase by treatment with $1 \mu \mathrm{M}$ tamoxifen for the indicated times (lanes 1-3). The cells were then restimulated to enter the cell cycle by addition of $500 \mathrm{nM}$ estradiol for the indicated times (lanes 4-6). Cell extracts were analyzed by immunoblotting with Ab's to the indicated proteins (top four panels) and for Cdk2 kinase activity assayed using histone $\mathrm{H} 1(\mathrm{HH} 1)$ as a substrate after immunoprecipitation of Cdk2 (bottom panel). (b) MCF-7 cells infected with retroviruses expressing the indicated proteins were either left asynchronous or were synchronized in G1 by depletion of estradiol and treated with tamoxifen for 48 or 96 hours, as indicated. The percentage of MCF-7 cells in S-phase was then analyzed by BrdU incorporation. The results shown in the first 10 bars are the mean percentage obtained from at least three independent experiments. 
in a subset of high-grade ductal carcinomas that are negative for ER expression and do not overexpress Her-2 (Figure 1). The inverse correlation between Skp2 and ER protein levels, as well as the direct correlation between Skp2 levels and tumor grade, was confirmed by immunohistochemistry in a second series of 84 breast cancers (sample set B) (Figure 2). Interestingly, our gene expression analysis also demonstrates that tumors overexpressing Skp2 are negative for keratins characteristic of epithelial luminal cells but show the expression of epithelial basal cell markers, including keratins 5 and 17 (Figure 1). A similar subtype of ER/Her-2-negative breast tumors with basal-like phenotype has been identified recently and found to be associated with poorer outcome as compared with the ER-positive subgroups of tumors with luminal phenotypes $(3,4)$. Accordingly, when we assessed the relationship between Skp2 and survival in sample set B, we found that high levels of Skp2 were associated with poor survival by univariate analysis. Larger series of tumors will have to be analyzed to determine the independent prognostic significance of Skp2 in breast cancer.

While in certain tumors p 27 is inactivated by cytoplasmic sequestration or by oncoproteins such as Myc and E1a through still not well-understood mechanisms, reduced protein levels of the cell cycle inhibitor p27 are frequently observed in a variety of human tumors and are associated with poor prognosis (reviewed in ref. 17). In breast carcinomas we and other investigators have shown previously that loss of p27 expression in tumor cells is an independent predictor of both overall survival and disease-free survival (28-30). In agreement with the role of Skp2 in p27 degradation, we found that Skp2 levels inversely correlate with p27 expression (Figure 2). An interesting finding was the fact that in a subset of breast cancers, Skp2 was expressed at low levels despite low expression of p27. This could be due to low levels but hyperactive Skp2 (e.g., because of mutations, overexpression of the Skp2-cofactor Cks1, etc.). Alternatively, in these tumors p27 downregulation could be due to other, as yet unknown, mechanisms. We also found that breast tumors expressing high Skp2 are characterized by high expression of markers of both S-phase and G2/M at both RNA and protein levels. In addition, we show that in both human cancers and breast cancer cells in culture, Skp2 protein is selectively expressed in a subset of proliferating cells (Figure 3). Remarkably, among the three different D-type cyclins, only cyclin D3 clustered with Skp2. These data confirm previous data showing positive correlation between cyclin D3 and high tumor grade (36). As expected, cyclin D1 was predominantly expressed in ER-positive, low-grade cancers (37). Interestingly, a recent study of gene expression profiling in breast cancer has identified genes regulating cell cycle to be one of the defining clusters of the molecular signature of poor prognosis (38).

Overall, our analysis in human tumors, taken together with the results present in the literature, demonstrate the existence of a subtype of high-grade, highly proliferating ER/Her-2-negative breast carcinomas with a basal-like phenotype characterized by high Skp2 and low p27 levels. These observations lead to the hypothesis that Skp2 overexpression plays a relevant role in the pathogenesis of this subset of tumors by providing a growth advantage, perhaps by promoting p 27 degradation. We investigated the oncogenic potential of Skp2 and found that, in contrast to normal epithelial cells, in breast cancer cells Skp2 expression is not altered by loss of adhesion to the cell matrix (Figure 4a). This finding suggests a role for Skp2 in the adhesion-independent ability of tumor cells to grow, and, in fact, inhibition of Skp2 activity reduces the adhesion-independent growth ability of cancer cells, as measured by the number of colonies in soft agar (Figure 4b). The effects produced by Skp2 inhibition are independent of the presence of the ER since they are observed both in ER-positive (MCF-7) and ER-negative cells (MDM-MB-435, which express very low levels of Her-2) (39).

Interestingly, while a specific therapy with anti-Her-2 Ab's (Herceptin) is currently used in ER-negative breast cancer that overexpresses Her-2 (2), no specific therapy has been identified in those tumors that are both ER and Her-2 negative. Since Skp2 expression is associated with lack of both ER and Her-2, and Skp2 inhibition affects the in vitro growth of ER/Her-2-negative breast cancer cells, our study identifies Skp2 as a potential specific therapeutic target in this subset of aggressive breast cancers. Finally, we show that enforced expression of Skp2 induces resistance to antiestrogens (Figure 5b). Albeit much less than in ER-negative tumors, where Skp2 is highly expressed in $61-67 \%$ of cases, Skp2 is also expressed at higher levels in 15\% (as detected by immunohistochemistry) to $23 \%$ (as detected by microarray) of the ER-positive tumors. Thus, it is tempting to speculate that Skp2 overexpression may be one of the factors implicated in the lack of response to hormonal therapy, which occurs in roughly one-third of tumors expressing ER. Similarly, it is possible to infer that deregulated Spk2 expression in ER-positive tumors treated with antihormonal therapy may play an important role in the development of resistance to antiestrogens.

In summary, our results support the notion that Skp2 is the product of a proto-oncogene and indicate that an alteration of Skp2 function and expression might contribute to the malignant behavior of breast tumors.

\section{Acknowledgments}

We thank Andrea Carrano, Alessia Montagnoli, and Alberto Ravaioli for their contribution to this work, and Myles Brown, Todd Golub, and Joanna Bloom for critical reading of the manuscript. $M$. Pagano is grateful to L. Yamasaki for continuous support. M. Pagano is recipient of the Irma T. Hirschl Scholarship, and L. Di Marcotullio of a fellowship from the American Italian Cancer Foundation. This work was supported by grants from the NIH (R01-CA76584 and R01-GM57587) and Department of Army (BC995695) to M. Pagano; NIH 
grants (R01-CA81755 and CA84995), a Novartis Investigator grant, Barr-Weaver and The Association for the Cure of Cancer of the Prostate (CaPCURE) awards to M. Loda; a National Cancer Institute Specialized Program of Research Excellence in Breast Cancer at Brigham \& Women's Hospital (CA89393) and a Breast Cancer Research Foundation Award to A. Richardson; a Hershey Prostate Cancer/Survivor Walk Award, a CaPCURE Award, and Department of the Army Grant (DAMD1701-1-0051) to S. Signoretti.

1. Muss, H.B. 2001. Role of adjuvant endocrine therapy in early-stage breast cancer. Semin. Oncol. 28:313-421.

2. Hortobagyi, G.N. 2001. Overview of treatment results with trastuzumab (Herceptin) in metastatic breast cancer. Semin. Oncol. 28:43-47.

3. Perou, C.M., et al. 2000. Molecular portraits of human breast tumours. Nature. 406:747-752.

4. Sorlie, T., et al. 2001. Gene expression patterns of breast carcinomas distinguish tumor subclasses with clinical implications. Proc. Natl. Acad. Sci. USA. 98:10869-10874.

5. Bai, C., et al. 1996. Skp1 connects cell cycle regulators to the ubiquitin proteolysis machinery through a novel motif, the F-box. Cell. 86:263-274.

6. Koepp, D., Harper, J.W., and Elledge, S.J. 1999. How the cyclin became a cyclin: regulated proteolysis in the cell cycle. Cell. 97:431-433.

7. Deshaies, R.J. 1999. SCF and Cullin/Ring H2-based ubiquitin ligases. Annu. Rev. Cell Dev. Biol. 15:435-467.

8. Kipreos, E., and Pagano, M. 2000. The F-box protein family. Genome Biol. 1:3002-3007.

9. Cenciarelli, C., et al. 1999. Identification of a human family of F-box proteins. Curr. Biol. 9:1177-1119.

10. Winston, J.T., Koepp, D.M., Zhu, C., Elledge, S.J., and Harper, J.W. 1999. A family of mammalian F-box proteins. Curr. Biol. 9:1180-1182.

11. Rubin, G.M., et al. 2000. Comparative genomics of the eukaryotes. Science. 287:2204-2215.

12. Zhang, H., Kobayashi, R., Galaktionov, K., and Beach, D. 1995. p19Skp-1 and p45Skp-2 are essential elements of the cyclin A-Cdk2 S phase kinase. Cell. 82:915-925.

13. Carrano, A.C., Eytan, E., Hershko, A., and Pagano, M. 1999. Skp2 is required for the ubiquitin-mediated degradation of the Cdk-inhibitor p27. Nat. Cell Biol. 1:193-199.

14. Sutterluty, H., et al. 1999. p45SKP2 promotes p27Kip1 degradation and induces S phase in quiescent cells. Nat. Cell Biol. 1:207-214.

15. Tsvetkov, L.M., Yeh, K.H., Lee, S., Sun, H., and Zhang, H. 1999. p27(Kip1) ubiquitination and degradation is regulated by the SCFSkp 2 complex through phosphorylated Thr187 in p27. Curr. Biol. 9:661-664.

16. Nakayama, K., et al. 2000. Targeted disruption of Skp2 results in accumulation of cyclin E and p27Kip1, polyploidy and centrosome overduplication. EMBOJ. 19:2069-2081.

17. Slingerland, J., and Pagano, M. 2000. Regulation of the cdk inhibitor p27 and its deregulation in cancer. J. Cell Physiol. 183:10-17.

18. Loda, M., et al. 1997. Increased proteasome-dependent degradation of the cyclin-dependent kinase inhibitor p27 in aggressive colorectal carcinomas. Nat. Med. 3:231-234.
19. Esposito, V., et al. 1997. Prognostic role of the cell cycle inhibitor p27 in non small cell lung cancer. Cancer Res. 57:3381-3385.

20. Kudo, Y., et al. 2001. High expression of S-phase kinase-interacting protein 2 , human F-box protein, correlates with poor prognosis in oral squamous cell carcinomas. Cancer Res. 61:7044-7047.

21. Chiarle, R., et al. 2000. Increased proteasome degradation of cyclin-dependent kinase inhibitor p27 is associated with a decreased overall survival in mantle cell lymphoma. Blood. 95:619-626.

22. Piva, R., et al. 1999. Proteasome-dependent degradation of p27/kip1 in gliomas. J. Neuropathol. Exp. Neurol. 58:691-696.

23. Carrano, A.C., and Pagano, M. 2001. Role of the F-box protein Skp2 in adhesion-dependent cell cycle progression. J. Cell Biol. 153:1381-1389.

24. Gstaiger, M., et al. 2001. Function of human Skp2 as an oncogene. Proc. Natl Acad. Sci. USA. 98:5043-5048.

25. Latres, E., et al. 2001. Role of the F-box protein Skp2 in lymphomagenesis. Proc. Natl Acad. Sci. USA. 98:2515-2520.

26. Hershko, D., et al. 2001. Inverse relationship between levels of p27 and its ubiquitin ligase subunit Skp2 in colorectal cancers. Cancer. 91:1745-1751.

27. Chiarle, R., et al. 2002. Skp2 expression in non-Hodgkins lymphomas inversely correlates with $\mathrm{p} 27$ expression and defines cells in S-phase. Am.J. Pathol. 160:1457-1466.

28. Catzavelos, C., et al. 1997. Decreased levels of the cell-cycle inhibitor p27Kip1 protein: prognostic implications in primary breast cancer. Nat. Med. 3:227-230.

29. Porter, P., et al. 1997. Expression of cell-cycle regulators p27Kip1 and cyclin $\mathrm{E}$, alone and in combination, correlate with survival in young breast cancer patients. Nat. Med. 3:222-225.

30. Tan, P., et al. 1997. The cell cycle inhibitor $\mathrm{p} 27$ is an independent prognostic marker in small (T1a,b) invasive breast carcinomas. Cancer Res. 57:1259-1263.

31. Golub, T.R., et al. 1999. Molecular classification of cancer: class discovery and class prediction by gene expression monitoring. Science. 286:531-537.

32. Signoretti, S., et al. 2000. Her-2-neu expression and progression toward androgen independence in human prostate cancer. J. Natl. Cancer Inst. 92:1918-1925.

33. Waltregny, D., et al. 2001. Androgen-driven prostate epithelial cell proliferation and differentiation in vivo involve the regulation of $\mathrm{p} 27 \mathrm{kip} 1$ through its proteasome-mediated degradation. Mol. Endocrinol. 15:765-782.

34. Tam, S.W., Theodoras, A.M., Shay,J.W., Draetta, G.F., and Pagano, M. 1994. Differential expression and regulation of cyclin D1 protein in normal and tumor human cells: association with Cdk4 is required for cyclin D1 function in G1 progression. Oncogene. 9:2663-2674.

35. Ohtsubo, M., Theodoras, A., Schumacher, J., Roberts, J.M., and Pagano, M. 1995. Human cyclin E, a nuclear protein essential for the G1-to S-phase transition. Mol. Cell. Biol. 15:2612-2124.

36. Wong, S.C., Chan, J.K., Lee, K.C., and Hsiao, W.L. 2001. Differential expression of $\mathrm{p} 16 / \mathrm{p} 21 / \mathrm{p} 27$ and cyclin D1/D3, and their relationships to cell proliferation, apoptosis, and tumour progression in invasive ductal carcinoma of the breast. J. Pathol. 194:35-42.

37. Shoker, B.S., et al. 2001. Immunodetectable cyclin D(1)is associated with oestrogen receptor but not Ki67 in normal, cancerous and precancerous breast lesions. Br.J. Cancer. 84:1064-1069.

38. van 't Veer, L.J., et al. 2002. Gene expression profiling predicts clinical outcome of breast cancer. Nature. 415:530-536.

39. Ueno, N.T., Yu, D., and Hung, M.C. 1997. Chemosensitization of HER$2 /$ neu-overexpressing human breast cancer cells to paclitaxel (Taxol) by adenovirus type 5 E1A. Oncogene. 15:953-960. 